\title{
Cost-effectiveness of CTA, MRA and DSA in patients with non-traumatic subarachnoid haemorrhage
}

\author{
Anna M. H. Sailer • Janneke P. Grutters • \\ Joachim E. Wildberger • Paul A. Hofman • \\ Jan T. Wilmink • Willem H. van Zwam
}

Received: 18 May 2013 / Accepted: 4 June 2013 / Published online: 10 July 2013

(C) The Author(s) 2013. This article is published with open access at Springerlink.com

\begin{abstract}
Objectives Intra-arterial digital subtraction angiography (DSA), magnetic resonance angiography (MRA) and computed tomographic angiography (CTA) are imaging modalities used for diagnostic work-up of non-traumatic subarachnoid haemorrhage. The aim of our study was to compare the cost-effectiveness of MRA, DSA and CTA in the first year after the bleed.

Methods A decision model was used to calculate costs and benefits (in quality-adjusted life-years [QALYs]) that accrued to cohorts of 1,000 patients. Costs and characteristics of diagnostic tests, therapy, patients' quality of life and associated costs were respected. The diagnostic strategy with highest QALYs and lowest costs was considered most cost-effective. Results DSA was the most effective diagnostic option, yielding on average 0.6039 QALYs (95\% CI, 0.5761-0.6327) per patient, followed by CTA 0.5983 QALYs $(95 \%$ CI, 0.5704 0.6278 ) and MRA 0.5947 QALYs (95\% CI, 0.56740.6237). Cost was lowest for DSA $(39,808 € ; 95 \% \mathrm{CI}$, $37,182-42,663)$, followed by CTA $(40,748 € ; 95 \% \mathrm{CI}$, $37,937-43,831)$ and MRA $(41,814 € ; 95 \%$ CI, 38,730$45,146)$. A strategy of CTA followed by DSA if CTA was negative or coiling deemed not feasible, was as effective as DSA alone at average costs of 39,767€ (95 \% CI, 36,90342,402).

Conclusion A combined strategy of CTA and DSA was found to be the most cost-effective diagnostic approach.
\end{abstract}

A. M. H. Sailer $(\bowtie) \cdot$ J. E. Wildberger • P. A. Hofman •

J. T. Wilmink · W. H. van Zwam

Department of Radiology, Maastricht University Medical Centre (MUMC), P. Debyelaan 25, 6229 HX Maastricht, The Netherlands e-mail: anni.sailer@mumc.nl

\section{J. P. Grutters}

Department of Epidemiology, Biostatistics and Health Technology

Assessment, Radbouw University Nijmegen Medical Centre,

Nijmegen, The Netherlands
Main Messages

- We defined a standard model for cost-effectiveness analysis in diagnostic imaging.

- Comparing total 1-year health costs and benefits, CTA is superior to MRA.

- A strategy of combining CTA and DSA was found to be the most cost-effective diagnostic approach.

Keywords Intracranial aneurysm - Cost effectiveness · Digital subtraction angiography $\cdot$ Magnetic resonance angiography $\cdot$ Computed tomography angiography

\section{Introduction}

Rupture of an intracranial aneurysm is a major cause of death and disability with an overall incidence of approximately 9 per 100,000 [1]. In the group of patients who survive the initial bleed, the risk of rebleeding without treatment is $1-2 \%$ per day for the first 4 weeks with a $70 \%$ case fatality rate $[2,3]$.

To prevent rebleeding from ruptured aneurysms, treatment is performed as soon as feasible in order to exclude the aneurysm from the arterial circulation $[2,4,5]$. Treatment options are either neurosurgical clipping via craniotomy or endovascular aneurysm occlusion by detachable platinum coils. The ISAT trial demonstrated better clinical outcome and significant risk reduction for endovascular treatment. Coiling is the preferred option in patients where both treatments are feasible [6-9]. Total treatment costs were found to be slightly higher for surgical clipping compared with endovascular coiling [10-13].

Non-traumatic subarachnoid haemorrhage (SAH) is caused in about $85 \%$ of patients by a ruptured intracranial aneurysm [14]. Diagnostic imaging is required to fulfil two tasks: (1) detect the presence of intracerebral aneurysms; (2) 
determine the most suitable treatment: endovascular coiling or surgical clipping.

DSA is the reference standard for detecting aneurysms and for determining the feasibility of coiling. Nevertheless this invasive and labour-intensive technique is relatively expensive and carries discomforts and potential risks [15-17]. Promising non-invasive and less costly diagnostic alternatives are computed tomographic angiography (CTA) and magnetic resonance angiography (MRA). CTA is easily available and can be rapidly performed in an acute setting. The main benefit of MRA is the fact that no ionising radiation is used.

Although costs and characteristics of diagnostic tests and treatment have been reported, we found no studies determining the optimal diagnostic pathway in patients with suspected ruptured intracranial aneurysms. Our study aimed to compare the cost-effectiveness of MRA, CTA and DSA in these patients. Cost-effectiveness of diagnostic tests in follow-up of coiled aneurysms has previously been evaluated by Schaafsma et al. [18].

\section{Materials and methods}

We previously assessed test characteristics and accuracy of MRA (1.5 T) and CTA in 75 patients with non-traumatic SAH [19]. Patients were recruited consecutively between 2004 and 2006, and for all patients MRA, CTA and DSA had been performed. Informed consent was given and this study protocol was approved by the Institutional Ethical Committee. DSA served as the standard of reference for aneurysm presence. Feasibility of coiling depended on the judgment of the interventionist based on DSA, or the actual suitability during a coiling attempt. For CTA and MRA two experienced neuroradiologists determined the presence of an aneurysm and judged suitability for endovascular treatment using common criteria, like dome-to-neck ratio and absence of branches originating from the base of the aneurysm. The observers were blinded to the results of other imaging examinations. Pooled sensitivity of CTA for the detection of aneurysms was $91.5 \%$ (95\% CI, 85.0-95.5) and specificity was $94.4 \%$ (95\% CI, 79.0-99.0). Pooled sensitivity of MRA for the detection of aneurysms was $95.4 \%$ (95\% CI, 89.8-98.1) and specificity was $83.3 \%$ (95 \% CI, 66.5-93.0). Pooled sensitivity of CTA in determining feasibility of endovascular coiling was $71.9 \%(95 \% \mathrm{CI}, 59.0-82.1)$ and specificity was $75.4 \%$ (95\% CI, 62.0-85.5). Pooled sensitivity of MRA in determining feasibility of endovascular coiling was $60.6 \%$ (95\% CI, 48.2-71.7) and specificity was $81.4 \%(95 \% \mathrm{CI}, 68.7-89.9)$. DSA as standard of reference was regarded to have a sensitivity and specificity for aneurysm detection and determination of best treatment of $100 \%$.

\section{Evaluation of costs and benefits: a decision tree}

A decision tree (using Excel Software) was developed to determine differences in health benefits and costs in patients with ruptured aneurysms for various diagnostic pathways [20]. For patients with acute non-traumatic subarachnoid haemorrhage, the decision tree delivers different health states due to characteristics of diagnostic imaging and choice of therapy. Health states were based on whether patients were alive and, when they were alive, whether they were well or disabled. Figure 1 shows the decision tree for MRA. A hypothetical cohort of patients passes through the model based on a 1-year period. More specifically, a cohort of patients with non-traumatic subarachnoid haemorrhage is divided over the different pathways in the decision tree, based on a set of probabilities that were derived from literature. This allowed us to synthesise evidence and thereby to evaluate health costs of diagnostic test and treatment as well as related quality of life and associated costs determined by diagnostic decision. A provider (or healthcare) perspective was used, which included only direct healthcare costs.

\section{Basic model and scenario model}

In the basic model, patients underwent DSA, MRA or CTA. Following our standard clinical practice, if no aneurysm was detected on CTA or MRA, an additional DSA study was performed. Patients without an intracranial aneurysm were not treated. In patients in whom an aneurysm was detected, feasibility of coiling of the aneurysm was determined. Depending on the result of each test, either a coiling or clipping procedure was performed. In patients where falsepositive feasibility of coiling was determined by CTA or MRA, angiography during the coiling procedure would show no feasibility and transfer to surgical clipping would have been performed. In case of false-negative determination of coiling, surgical clipping would have been performed, although coiling would have been feasible.

In a scenario analysis we explored two alternative strategies. CTA and MRA are less expensive and less invasive than DSA. However, they may incorrectly characterise an aneurysm as not suitable for coiling. We therefore analysed whether it is cost-effective to add DSA to the CTA and MRA strategies only if an aneurysm is deemed not suitable for endovascular treatment. In this scenario, in each patient in whom an aneurysm was detected which was deemed not suitable for coiling by MRA or CTA, an additional DSA study was considered to be performed.

\section{Model parameters}

Sensitivity and specificity of the diagnostic tests for detection of aneurysms and determination of treatment possibility were taken as input parameters to the decision model. For the 


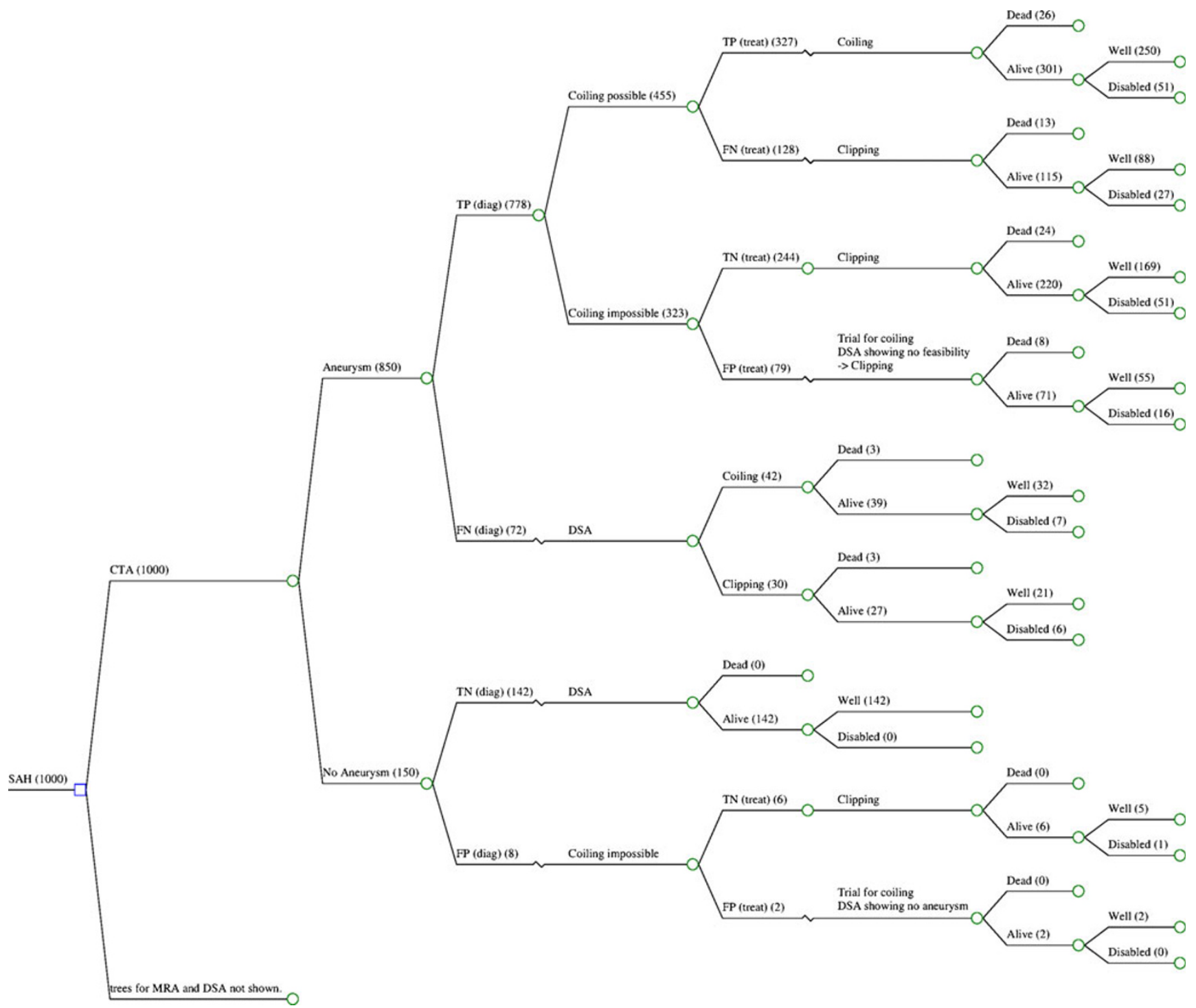

Fig. 1 Markov decision tree basic model showing exemplary branch for CTA. Branches for MRA and DSA not shown in detail. TP truepositive, $F N$ false-negative, $T N$ true-negative, $F P$ false-positive test

costs of DSA, MRA and CTA, standard prices from the Dutch manual for cost research were used [21]. These include expenditures for personnel, equipment, materials, maintenance, housing, cleaning, administration and overheads. Total 1-year costs of surgical clipping and endovascular coiling were derived from a literature search for western countries [10]. Health outcome after 1 year of treatment was derived from the ISAT trial $[6,7]$.

Input parameters for related utilities [22] and costs [18, $21,23]$, as well as health risk of DSA, are based on available literature [17, 24]. All costs were updated to 2010 by means of national price index figures [25] and expressed in euros $(1 €=\$ 1.32)$. Standard discount rates of $1.5 \%$ for effects and $4 \%$ for costs according to Dutch guidelines were used [21]. Table 1 shows the model input parameters and their sources. result. Example of absolute patient numbers from base case analysis in brackets. Input parameters derived from Table 1

Model assumptions

We assumed that DSA, as the standard of reference, has a sensitivity and specificity of $100 \%$ in detecting aneurysms and determining feasibility of coiling. Furthermore, we assumed no significant gender- or age-related differences in outcome, as well as no influence due to aneurysm size or location. Patients presenting with acute $\mathrm{SAH}$ without presence of a ruptured aneurysm were assumed to have no other intracranial vascular pathology to be treated.

Data analysis

In our model, we evaluated the outcome of diagnostic pathways based on the input parameters in Table 1. In the base case 
Table 1 Model input parameters

Model parameter

Discount rates

cDR (cost discount rate)

oDR (outcome discount rate)

Treatment independent parameters

Probabilities, (p)

pAneurysm in case of non-traumatic SAH

Costs, c (€)

Diagnostics

cDSA

cMRA

cCTA

Treatment

cCoiling

cClipping

Health state costs

Number of days in nursing home per year (d)

Costs per day in nursing home (euros/d)

cDisability

Event costs

cDeath

Utilities (u)

uWell

uWell after SAH

uDisabled

uDead

Clinical outcome probabilities

Probability of being well after clipping

Probability of being disabled after clipping

pClipdead (probability of being dead after clipping)

pClipwell (probability of being well after survived clipping)

Probability of being well after coiling

Probability of being disabled after coiling

pCoildead (probability of being dead after coiling)

pCoilwell (probability of being well after survived coiling)

Probability of being well after DSA

Probability of being disabled after DSA

pDSAdead (probability of being dead after DSA)

pDSAwell (probability of being well after survived DSA)

Probability of being dead/disabled after surgery without aneurysm

pSurgdead

pSurgdisab

pCoilTP (probability coiling is feasible in true-positive aneurysm)

pCoilFP (probability coiling is feasible in false-positive aneurysm)

Treatment dependent parameters

Probabilities

pMRAtpD (sensitivity of MRA in diagnosing aneurysm)

pMRAtnD (specificity of MRA in diagnosing aneurysm)

pCTAtpD (sensitivity of CTA in diagnosing aneurysm)

pCTAtnD (specificity of CTA in diagnosing aneurysm)
Mean

$4 \%$

$1.5 \%$

0.85

$725 €$

$252 €$

$197 €$

$38,238 €$

$31,739 €$

$1,833 €$

$2,503 €$

365

$241 €$

$87,975 €$

$2,741 €$

0.78

0.72

0.25

0.00

0.69

0.21

0.10

0.77

0.76

0.16

0.08

0.83

0.998

0.002

0.000

0.998

0.025

0.132

0.585

0

Value

0.954

0.833

0.915

0.944

$$
0.019
$$

0.001

0.004

0.008
0.018
0.061
0.024

\section{$\mathrm{SE} / \mathrm{SD} /$ range $^{\mathrm{a}}$}

Distribution

Source

fixed

fixed

fixed

fixed

fixed

fixed

gamma

gamma

beta

[18, 22]

0.65-0.80

$0.21-0.30$

triangular

$[18,22]$

triangular

$[18,22]$

fixed

$[18,22]$

beta

[7]

beta

[7]

[7]

[7]

[7]

[7]

[7]

[7]

$[17,24]$

beta

$[17,24]$

beta

$[17,24]$

fixed

beta

[30]

beta

beta

[19]

fixed

[19]

$\mathrm{SE} / \mathrm{SD} /$ range $^{\mathrm{a}}$

Distribution

Source

beta

[19]

beta

[19]

beta

[19]

[19] 


\begin{tabular}{|c|c|c|c|c|}
\hline pMRAtpT (sensitivity of MRA in determining whether coiling is feasible) & 0.606 & 0.058 & beta & $\mathrm{b}$ \\
\hline pMRAtnT (specificity of MRA in determining whether coiling is feasible) & 0.814 & 0.050 & beta & $\mathrm{b}$ \\
\hline pCTAtpT (sensitivity of CTA in determining whether coiling is feasible) & 0.719 & 0.056 & beta & b \\
\hline pCTAtnT (specificity of CTA in determining whether coiling is feasible) & 0.754 & 0.057 & beta & $\mathrm{b}$ \\
\hline pDSAtpD (sensitivity of DSA in diagnosing aneurysm) & 1 & & fixed & [19] \\
\hline pDSAtnD (Specificity DSA in diagnosing aneurysm) & 1 & & fixed & [19] \\
\hline pDSAtpT (sensitivity of DSA in determining whether coiling is feasible) & 1 & & fixed & [19] \\
\hline pDSAtnT (specificity of DSA in determining whether coiling is feasible) & 1 & & fixed & [19] \\
\hline
\end{tabular}

${ }^{a}$ Range presented for triangular distributions

${ }^{\mathrm{b}}$ Data based on study [19]

analysis, the average costs and effects were calculated for a hypothetical cohort of 1,000 patients. We compared total 1-year costs of diagnostic test, treatment option and health state to 1year health benefits in terms of quality-adjusted life-years (QALYs). The alternative that yielded the highest number of QALYs was considered to be most effective. The current analysis was performed from a decision-analytic perspective. This implies that for an intervention to be adopted, it has to be cost-effective compared with its next best alternative [26]. If a strategy is less costly and more effective, it is superior to and dominates the alternative strategy. If a strategy is more costly and less effective than its alternative, it is dominated by the alternative. In case the strategy is more costly and more effective, or less costly and less effective than the alternative, incremental cost-effectiveness ratios (ICERs) were calculated from each model by dividing the incremental costs by the incremental QALYs. The decision whether the strategy is deemed costeffective then depends on how much society is willing to pay for a QALY gained. In The Netherlands the informal societal willingness to pay (WTP) threshold level is $80,000 €$ (\$ 106,000) [27]. If the ICER is lower than this level, we conclude that the strategy is cost-effective compared with the alternative. Additionally, a scenario analysis was performed in which, for all patients, DSA was performed in case an aneurysm was visible but coiling was deemed not feasible.

\section{Sensitivity analysis}

Uncertainty regarding the model input parameters was explored with a probabilistic sensitivity analysis using Monte Carlo simulation [20, 28]. For this purpose we assigned distributions to all uncertain parameter. With Monte Carlo simulation, 1,000 different samples were taken from these parameter distributions. For each sample, the hypothetical cohort runs through the model based on these sampled probabilities, and costs and effects are derived. This results in 1,000 estimates of costs and effects for the hypothetical cohort, representing the uncertainty in the cost-effectiveness estimation [18]. All assigned distributions are listed in Table 1.
To illustrate the results of the simulation, cost-effectiveness acceptability curves (CEACs) were calculated [29]. CEACs show the probability that a strategy is cost-effective, given different values of willingness to pay for a QALY.

Additionally, univariate sensitivity analyses were performed for test characteristics and costs of DSA, MRA and CTA, as well as for costs of coiling and clipping, to determine association between these model input parameters and cost-effectiveness outcome.

\section{Results}

\section{Basic model}

Comparing expected average 1-year health benefits, DSA was the most effective diagnostic option, yielding 0.6039 QALYs (95\% CI, 0.5761-0.6327), followed by CTA yielding 0.5983 QALYs (95\% CI, 0.5704-0.6278) and MRA yielding 0.5947 QALYs (95\% CI, 0.5674-0.6237). The total expected 1-year health costs were lowest for DSA $(39,808 € ; 95 \%$ CI, 37,18242,663), followed by CTA (40,748 €; $95 \%$ CI, 37,937-43,831) and MRA (41,814€; $95 \%$ CI, 38,730-45,146). Initial diagnostic costs were highest for DSA, while therapy and health staterelated costs were lowest for DSA, resulting in DSA being the least costly diagnostic option. CTA as diagnostic test resulted in 128 out of 1,000 patients being referred for clipping although coiling would have been feasible. With MRA, 187 patients out of 1,000 were treated surgically, although endovascular treatment would have been feasible. DSA was both more effective and less costly and dominated CTA and MRA. CTA was more effective and less costly than MRA and thus dominated MRA. Table 2 shows costs and QALYs for the three diagnostic tests.

\section{Scenario model}

The strategy that included DSA for every patient for whom coiling was not deemed feasible on the basis of CTA and MRA, led to equal effectiveness for DSA, MRA and CTA (0.6039 QALYs each). In this approach no patient was 
Table 2 Related 1-year costs and QALYs for MRA, CTA and DSA per patient in the basic model

\begin{tabular}{|c|c|c|c|c|c|c|}
\hline & \multicolumn{2}{|l|}{ MRA } & \multicolumn{2}{|l|}{ CTA } & \multicolumn{2}{|l|}{ DSA } \\
\hline & Costs $(€)$ & QALYs & Costs $(€)$ & QALYs & Costs $(€)$ & QALYS \\
\hline MRA resp. CTA & 252 & 0.5947 & 197 & 0.5983 & & 0.6039 \\
\hline DSA & $168^{\mathrm{a}}$ & & $214^{\mathrm{a}}$ & & 725 & \\
\hline Total diagnostic & 420 & & 411 & & 725 & \\
\hline Treatment & 26,734 & & 26,120 & & 25,553 & \\
\hline Death & 217 & & 212 & & 205 & \\
\hline Disability costs & 14,443 & & 14,005 & & 13,325 & \\
\hline Total & 41,814 & 0.5947 & 40,748 & 0.5983 & 39,808 & 0.6039 \\
\hline
\end{tabular}

${ }^{a}$ Average costs per patient for DSA performed in case CTA/MRA showing no aneurysm

treated surgically unless coiling was no option. In this scenario, CTA was estimated less costly, reaching a total average 1-year cost per patient of $39,767 €$, and therefore costeffective compared with MRA and DSA, which reached costs of 39,851 $€$ and 39,808 $€$ respectively. Table 3 shows total expected 1-year costs and QALYs as well as incremental costs and QALYs for diagnostic tests in the scenario analysis and basic model.

Sensitivity analysis

In univariate sensitivity analyses we explored whether changing individual parameters altered the conclusions of our base case analysis. Assuming equal treatment cost for coiling and clipping did not change the conclusions. Furthermore, results remained stable for the assumption of higher costs of DSA up to factor 2.8. Higher sensitivity and specificity for detection of aneurysms and determination of feasibility of coiling for CTA and MRA up to $96 \%$ or a reduction of sensitivity and specificity for DSA to $90 \%$ also yielded stable results. In the scenario analysis, the strategy where CTA is followed by DSA remained the least costly strategy for a sensitivity of determination of coiling within the range of $60-100 \%$. The probabilistic sensitivity analysis showed that in the basic model analysis, DSA had a 98-100\% probability of being cost-effective. In the scenario analysis, CTA had the highest probability of being cost-effective ( $91 \%$ ), followed by DSA (9\%) and MRA (0\%). Because in the scenario analysis effectiveness is equal for all strategies, CEACs are constant over the different values of willingness to pay for a QALY.

\section{Discussion}

When a patient presents with a suspected ruptured intracranial aneurysm, imaging is a challenge. Due to high risk of case fatality in ruptured intracranial aneurysms, timely detection of a causative aneurysm and determination of appropriate treatment are mandatory. Based on imaging results, patients may be left untreated, or their aneurysms clipped surgically, or they receive endovascular treatment. In recent years, much research has been done to evaluate the best treatment options and to optimise the approach to imaging modalities. In particular, the role and necessity of DSA in this patient population has been widely discussed [31-34]. In our study we compared the diagnostic work-up by CTA, MRA and DSA based on a cost-effectiveness approach. We found that DSA is more cost-effective than CTA, which is in turn superior to MRA. A combination strategy of CTA followed by DSA if endovascular treatment is deemed not feasible was found to be as effective as DSA alone at slightly lower expected costs.

Our analysis is based on a 1-year horizon. This holds potential conflict concerning the long-term validity of the results. Probabilities of health outcome which we clustered from the ISAT trial are relatively stable in the long run and do not change our results [8]. Incomplete occlusion and refilling of the aneurysm is a complication occurring mainly in coiling which can cause rebleeding, resulting in a higher rate of follow-up imaging costs and re-interventions in the endovascular group. The rate of recanalisation is highest in the first months and decreases significantly over time [35-38]. Follow-up for detection of recanalisation after 6 months may not be necessary [36]. Wolstenholme et al. [10] determined cost of coiling and clipping in the first year for western countries. Calculation of total costs for both treatment options included not only cost for material, intensive care and standard unit days and medication but also cost of follow-up imaging and re-intervention for the first year. Taking the results from literature, we can assume that the validity of our data will be stable for the following years.

Our basic model results show that DSA is the most costeffective imaging modality and is superior to both MRA and CTA. Although initial test costs are about three times higher than those of MRA and CTA, optimal detection of aneurysms and determination of treatment in DSA yield lower overall 1- 
Table 3 Total one-year outcome and incremental cost-effectiveness ratio (ICER) for basic and scenario model

\begin{tabular}{|c|c|c|c|c|c|c|}
\hline & Costs $(€)$ & $(95 \% \mathrm{CI})$ & QALYs & $(95 \% \mathrm{CI})$ & & \\
\hline \multicolumn{7}{|c|}{ Basic model } \\
\hline DSA & 39,808 & $(37,182 ; 42,663)$ & 0.6039 & $(0.5761 ; 0.6327)$ & & \\
\hline CTA & 40,748 & $(37,937 ; 43,831)$ & 0.5983 & $(0.5704 ; 0.6278)$ & & \\
\hline MRA & 41,814 & $(38,730 ; 45,146)$ & 0.5947 & $(0.5674 ; 0.6237)$ & & \\
\hline \multicolumn{7}{|c|}{ Scenario model } \\
\hline DSA & 39,808 & $(36,982 ; 42,414)$ & 0.6039 & $(0.5771 ; 0.6333)$ & & \\
\hline CTA & 39,767 & $(36,903 ; 42,402)$ & 0.6039 & $(0.5771 ; 0.6333)$ & & \\
\hline \multirow[t]{2}{*}{ MRA } & 39,851 & $(37,003 ; 42,486)$ & 0.6039 & $(0.5771 ; 0.6333)$ & & \\
\hline & Incremental costs $(€)$ & $(95 \% \mathrm{CI})$ & Incremental QALYs & $(95 \% \mathrm{CI})$ & ICER ( $€$ per QALY) & Comparator \\
\hline \multicolumn{7}{|c|}{ Basic model } \\
\hline \multicolumn{7}{|c|}{ DSA } \\
\hline CTA & 940 & $(10 ; 2,122)$ & -0.006 & $(-0.003 ;-0.009)$ & Dominated & DSA \\
\hline MRA & 2,007 & $(604 ; 3,767)$ & -0.009 & $(-0.005 ;-0.015)$ & Dominated & DSA \\
\hline \multicolumn{7}{|c|}{ Scenario model } \\
\hline \multicolumn{7}{|c|}{ DSA } \\
\hline CTA & -40 & $(-103 ; 20)$ & 0.000 & $(0.000 ; 0.000)$ & Dominant & DSA \\
\hline MRA & 84 & $(-15 ; 98)$ & 0.000 & $(0.000 ; 0.000)$ & Dominated & CTA \\
\hline
\end{tabular}

CI Confidence Interval (calculated based on the probabilistic sensitivity analysis)

year costs per patient and the highest QALYs. The morbidity of DSA, although low, was included as an input parameter in our model $[17,24]$. Nevertheless, we did not consider the potential influence of test-related short-term disutility described by Swan et al. [39]. Depending on the severity grade of clinical presentation, the short-term discomfort undergoing DSA experienced by patients with SAH might be of varying importance and influence to our model might be less compared with in elective imaging.

We assumed DSA to be the standard of reference with sensitivity and specificity for detection and determination of treatment of $100 \%$. It is debatable whether sensitivity of DSA to cerebral aneurysms is $100 \%$ in an acute setting [40]. When coiling was performed, costs for diagnostic DSA were accounted for separately. Costs of imaging were estimated at $725 €$, following the Dutch costing guideline [21]. Actual costs of DSA might be higher than in our model and likely differ in other countries. We therefore performed sensitivity analysis showing stable results for assumption of $90 \%$ sensitivity and specificity in aneurysm detection and coilability, as well as costs up to $2,025 €$ for DSA. Given this range of diagnostic performance and costs, other researchers or decision-makers can assess transferability of the results to their specific situation and jurisdiction [41]. Differences between United States cost estimates and European cost estimates, in particular, may not change conclusions, as costs are proportionally higher in the United States [42].

The test characteristics for CTA and MRA are based on our study performed with 75 patients. In literature, meta-analyses of test characteristics of CTA as well as other studies show higher sensitivity and specificity for CTA and MRA in the detection of aneurysms and determination of treatment compared with our study [43-45]. Since our basic model results are stable in sensitivity analysis with assumption of $96 \%$ sensitivity and specificity for aneurysm detection as well as determination of treatment for CTA and MRA, we can assume that our conclusion is valid in a broad range of diagnostic performance. In the scenario model sensitivity analysis, increasing sensitivity and specificity for CTA and MRA shows even increasing preference of our imaging strategy starting with non-invasive imaging compared with only DSA.

Comparing the two non-invasive imaging modalities, CTA dominated MRA in the basic model and thus was superior. This was mainly due to lower sensitivity in determination of feasibility of coiling in MRA. Beside its inferiority in cost-effectiveness, MRA has limitations as first line imaging in acute settings, as it might be unavailable during night hours and not applicable in severe clinical presentation.

Our scenario model with initial CTA followed by DSA in case an aneurysm is detected but deemed not suitable for coiling was found to be the most cost-effective imaging strategy overall. The slightly lower costs compared with only DSA result from the group of patients where on CTA an aneurysm is detected which is deemed feasible for coiling. In this group endovascular treatment will be initiated without previous diagnostic DSA. Nevertheless comparing these two approaches, the average reduction in costs per patient is $40 €$, which is marginal in comparison to the overall 1-year costs. In case of suspected SAH, standard diagnostic imaging is non-contrast enhanced CT (NECT), followed by examination of CSF, obtained by lumbar puncture, if NECT is negative [46]. In case a SAH is detected on NECT, performing an additional CTA study can be assumed to be less expensive than the total CTA cost that we used as an input parameter in our model. In fact only additional contrast application would need to be accounted for. Considering this, we might assume that the 
actual cost advantage of the scenario strategy will likely be higher.

There are heated discussions about whether or not to always perform DSA in patients with suspected ruptured intracranial aneurysms [47-56]. Our study contributes to this discussion with two important findings. First, DSA is both more effective, yielding higher QALYs, and less costly in overall costs than CTA and MRA. DSA is therefore superior to the non-invasive imaging modalities and should play a key role in diagnostic work-up of non-traumatic SAH. Second, we analysed a scenario that combines invasive and non-invasive diagnostic tests. We found the less costly and therefore most efficient diagnostic approach overall to be a strategy, starting with CTA as the first examination, followed by DSA if coiling is not deemed feasible. This means that every patient undergoes additional DSA unless an aneurysm has been detected and deemed suitable for endovascular coiling. This strategy yields the same amount of QALYs compared with DSA alone in all patients, but results in lower average costs per patient. In comparison to diagnostic DSA, CTA is easily available during night hours and can directly be performed on hospital admission. This further emphasises CTA as a first-line imaging strategy, as it may fasten clinical treatment decisions and therefore help avoid rebleedings.

Besides representing the most cost-effective approach overall, we believe that the strategy of combining CTA and DSA also represents the most practical approach in routine clinical practice. For patients with suspected SAH, we therefore recommend performing a non-contrast enhanced CT scan first. If a SAH is demonstrated, CTA is directly performed. When CTA shows no aneurysm or shows an aneurysm which is not suitable for coiling, an additional DSA study should be performed.

\section{Conclusions}

For diagnostic work-up of suspected ruptured intracranial aneurysms, a combined strategy of CTA followed by DSA if CTA is negative or endovascular treatment deems not feasible is found to be as the most cost-effective approach overall.

\section{Conflict of interests None.}

Open Access This article is distributed under the terms of the Creative Commons Attribution License which permits any use, distribution, and reproduction in any medium, provided the original author(s) and the source are credited.

\section{References}

1. de Rooij NK, Linn FH, van der Plas JA, Algra A, Rinkel GJ (2007) Incidence of subarachnoid haemorrhage: a systematic review with emphasis on region, age, gender and time trends. J Neurol Neurosurg Psychiatry 78:1365-1372

2. Connolly ES Jr, Rabinstein AA, Carhuapoma JR et al (2012) Guidelines for the management of aneurysmal subarachnoid haemorrhage: a guideline for healthcare professionals from the American Heart Association/ American Stroke Association. Stroke 43:1711-1737

3. Starke RM, Connolly ES Jr (2011) Rebleeding after aneurysmal subarachnoid haemorrhage. Neurocrit Care 15:241-246

4. de Gans K, Nieuwkamp DJ, Rinkel GJ, Algra A (2002) Timing of aneurysm surgery in subarachnoid haemorrhage: a systematic review of the literature. Neurosurgery 50:336-340, discussion 340-342

5. Weil AG, Zhao JZ (2012) Treatment of ruptured aneurysms: earlier is better. World Neurosurg 7:263-265

6. Molyneux A, Kerr R, International Subarachnoid Aneurysm Trial (ISAT) Collaborative Group (2002) International Subarachnoid Aneurysm Trial (ISAT) of neurosurgical clipping versus endovascular coiling in 2143 patients with ruptured intracranial aneurysms: a randomised trial. Lancet 360:1267-1274

7. Molyneux AJ, Kerr RS, Yu LM et al (2005) International subarachnoid aneurysm trial (ISAT) of neurosurgical clipping versus endovascular coiling in 2143 patients with ruptured intracranial aneurysms: a randomised comparison of effects on survival, dependency, seizures, rebleeding, subgroups, and aneurysm occlusion. Lancet 366:809-817

8. Klompenhouwer EG, Dings JT, van Oostenbrugge RJ, Oei S, Wilmink JT, van Zwam WH (2011) Single-center experience of surgical and endovascular treatment of ruptured intracranial aneurysms. AJNR Am J Neuroradiol 32:570-575

9. van der Schaaf I, Algra A, Wermer M et al (2005) Endovascular coiling versus neurosurgical clipping for patients with aneurysmal subarachnoid haemorrhage. Cochrane Database Syst Rev 19, CD003085

10. Wolstenholme J, Rivero-Arias O, Gray A et al (2008) Treatment pathways, resource use, and costs of endovascular coiling versus surgical clipping after aSAH. Stroke 39:111-119

11. Lawson MF, Hoh BL (2010) Clipping versus coiling: the total hospital cost of aneurysm treatment. World Neurosurg 73:430-431

12. Hoh BL, Chi YY, Lawson MF, Mocco J, Barker FG (2010) Length of stay and total hospital charges of clipping versus coiling for ruptured and unruptured adult cerebral aneurysms in the Nationwide Inpatient Sample Database 2002 to 2006. Stroke 41:337-342

13. Bairstow P, Dodgson A, Linto J, Khangure M (2002) Comparison of cost and outcome of endovascular and neurosurgical procedures in the treatment of ruptured intracranial aneurysms. Australas Radiol 46:249-251

14. van Gijn J, Kerr RS, Rinkel GJ (2007) Subarachnoid haemorrhage. Lancet 369:306-318

15. Kaufmann TJ, Huston J 3rd, Mandrekar JN, Schleck CD, Thielen KR, Kallmes DF (2007) Complications of diagnostic cerebral angiography: evaluation of 19,826 consecutive patients. Radiology 243:812-819

16. Fifi JT, Meyers PM, Lavine SD et al (2009) Complications of modern diagnostic cerebral angiography in an academic medical center. J Vasc Interv Radiol 20:442-447

17. Willinsky RA, Taylor SM, TerBrugge K, Farb RI, Tomlinson G, Montanera W (2003) Neurologic complications of cerebral angiography: prospective analysis of 2,899 procedures and review of the literature. Radiology 227:522-528

18. Schaafsma JD, Koffijberg H, Buskens E, Velthuis BK, van der Graaf Y, Rinkel GJE (2010) Cost-effectiveness of magnetic resonance angiography versus intra-arterial digital subtraction angiography to follow-up patients with coiled intracranial aneurysms. Stroke 41:1736-1742

19. van Zwam HW, Hofman PM, Kessels AG, Oei TK, Heijboer RJ, Wilmink JT (2012) Diagnostic performance of contrast enhanced 
magnetic resonance angiography in detecting intracranial aneurysms in patients presenting with subarachnoid haemorrhage. EJMINT 1240000078. Available via http://www.ejmint.org/original-article/ 1240000078. Accessed March 102013

20. Briggs A, Sculpher M (1998) An introduction to Markov modelling for economic evaluation. PharmacoEconomics 13:397-409

21. Hakkaart-van Roijen L, Tan SS, Bouwmans CAM (2010) Manual for costing research. Dutch Health Care Insurance Board, Amstelveen

22. Post PN, Stiggelbout AM, Wakker PP (2001) The utility of health states after stroke: a systematic review of the literature. Stroke 32:1425-1429

23. Buskens E, Nederkoorn PJ, Buijs-Van Der Woude T et al (2004) Imaging of carotid arteries in symptomatic patients: costeffectiveness of diagnostic strategies. Radiology 233:101-112

24. Cloft HJ, Joseph GJ, Dion JE (1999) Risk of cerebral angiography in patients with subarachnoid haemorrhage, cerebral aneurysm, and arteriovenous malformation: a meta-analysis. Stroke 30:317-320

25. Statline, Available from http://statline.cbs.nl. Access date 05/02/2013

26. Claxton K, Sculpher M, Drummond M (2002) A rational framework for decision making by the National Institute for Clinical Excellence (NICE). Lancet 360:711-715

27. Council for Public Health and Health Care to the Minister of Health, Welfare and Sport (2006) Sensible and sustainable care. Recommendations produced by the Council for Public Health and Health Care to the Minister of Health, Welfare and Sport, The Hague. Available via http://www.rvz.net/uploads/ docs/Sensible_and_sustainable_care.pdf. Accessed 10 February 2013

28. Ades AE, Claxton K, Sculpher M (2006) Evidence synthesis, parameter correlation and probabilistic sensitivity analysis. Health Econ 15:373-381

29. van Hout BA, Al MJ, Gordon GS, Rutten FF (1994) Costs, effects and C/E-ratios alongside a clinical trial. Health Econ 3:309-339

30. Higashida RT, Lahue BJ, Torbey MT, Hopkins LN, Leip E, Hanley DF (2007) Treatment of unruptured intracranial aneurysms: a nationwide assessment of effectiveness. AJNR Am J Neuroradiol 28:146-151

31. Moran CJ (2011) Aneurysmal subarachnoid haemorrhage: DSA versus CT angiography - is the answer available? Radiology 258:15-17

32. Agid R, Willinsky RA, Farb RI, Terbrugge KG (2008) Life at the end of the tunnel: why emergent CT angiography should be done for patients with acute subarachnoid haemorrhage. AJNR Am J Neuroradiol 29:e45, author reply e46-47

33. Fox AJ, Symons SP, Aviv RI (2008) CT angiography is state-of-theart first vascular imaging for subarachnoid haemorrhage. AJNR Am J Neuroradiol 29:e41-e42, author reply e46-47

34. Livingston RR (2008) Regarding the risk of death from CT angiography in patients with subarachnoid haemorrhage. AJNR Am J Neuroradiol 29:e44, author reply e46-47

35. Ries T, Siemonsen S, Thomalla G, Grzyska U, Zeumer H, Fiehler J (2007) Long-term follow-up of cerebral aneurysms after endovascular therapy prediction and outcome of retreatment. AJNR Am J Neuroradiol 28:1755-1761

36. Sluzewski M, van Rooij WJ, Rinkel GJ, Wijnalda D (2003) Endovascular treatment of ruptured intracranial aneurysms with detachable coils: long-term clinical and serial angiographic results. Radiology 227:720-724

37. Raymond J, Guilbert F, Weill A et al (2003) Long-term angiographic recurrences after selective endovascular treatment of aneurysms with detachable coils. Stroke 34:1398-1403

38. Sprengers ME, Schaafsma J, van Rooij WJ et al (2008) Stability of intracranial aneurysms adequately occluded 6 months after coiling: a 3T MR angiography multicenter long-term follow-up study. AJNR Am J Neuroradiol 29:1768-1774

39. Swan JS, Langlotz CP (1998) Patient preference for magnetic resonance versus conventional angiography: assessment methods and implications for cost-effectiveness analysis - an overview. Invest Radiol 33:553-559

40. DeLaPaz RL, Wippold FJ 2nd, Cornelius RS et al (2011) ACR Appropriateness Criteria ${ }^{\circledR}$ on cerebrovascular disease. J Am Coll Radiol 8:532-538

41. Welte R, Feenstra T, Jager H, Leidl R (2008) A decision chart for assessing and improving the transferability of economic evaluation results between countries. PharmacoEconomics 228:857-876

42. Hunink MG (2008) Cost-effectiveness analysis: some clarifications. Radiology 249:753-755

43. Menke J, Larsen J, Kallenberg K (2011) Diagnosing cerebral aneurysms by computed tomographic angiography: meta-analysis. Ann Neurol 69:646-654

44. Westerlaan HE, van Dijk MJ, Jansen-van der Weide MC et al (2011) Intracranial aneurysms in patients with subarachnoid haemorrhage: CT angiography as a primary examination tool for diagnosis-systematic review and meta-analysis. Radiology 258:134-145

45. Chen YC, Sun ZK, Li MH et al (2012) The clinical value of MRA at 3.0 $\mathrm{T}$ for the diagnosis and therapeutic planning of patients with subarachnoid haemorrhage. Eur Radiol 22:1404-1412

46. Bederson JB, Connolly ES Jr, Batjer HH et al (2009) Guidelines for the management of aneurysmal subarachnoid haemorrhage: a statement for healthcare professionals from a special writing group of the Stroke Council, American Heart Association. Stroke 40:994 1025

47. Kallmes DF, Layton K, Marx WF, Tong F (2007) Death by nondiagnosis: why emergent $\mathrm{CT}$ angiography should not be done for patients with subarachnoid haemorrhage. AJNR Am J Neuroradiol 28:1837-1838

48. Westerlaan HE, Gravendeel J, Fiore D et al (2007) Multislice CT angiography in the selection of patients with ruptured intracranial aneurysms suitable for clipping or coiling. Neuroradiology 49:997-1007

49. Dehdashti AR, Rufenacht DA, Delavelle J, Reverdin A, de Tribolet N (2003) Therapeutic decision and management of aneurysmal subarachnoid haemorrhage based on computed tomographic angiography. Br J Neurosurg 17:46-53

50. González-Darder JM, Pesudo-Martínez JV, Feliu-Tatay RA (2001) Microsurgical management of cerebral aneurysms based in CT angiography with three-dimensional reconstruction (3D-CTA) and without preoperative cerebral angiography. Acta Neurochir (Wien) 143:673-679

51. Chen W, Yang Y, Xing W, Qiu J, Peng Y (2010) Application of multislice computed tomographic angiography in diagnosis and treatment of intracranial aneurysms. Clin Neurol Neurosurg 112:563-571

52. Papke K, Kuhl CK, Fruth M et al (2007) Intracranial aneurysms: role of multidetector CT angiography in diagnosis and endovascular therapy planning. Radiology 244:532-540

53. Villablanca JP, Achiriolaie A, Hooshi P et al (2005) Aneurysms of the posterior circulation: detection and treatment planning using volume-rendered three-dimensional helical computerized tomography angiography. J Neurosurg 103:1018-1029

54. Agid R, Lee SK, Willinsky RA, Farb RI, terBrugge KG (2006) Acute subarachnoid haemorrhage: using 64-slice multidetector CT angiography to "triage" patients' treatment. Neuroradiology 48:787-794

55. Jayaraman MV, Haas RA, Do HM, Meyers PM (2010) Should CT angiography be routinely used in patients suspected of having aneurysmal subarachnoid haemorrhage? No! Radiology 254:314-315

56. Kaufmann TJ, Kallmes DF (2008) Diagnostic cerebral angiography: archaic and complication-prone or here to stay for another 80 years? AJR Am J Roentgenol 190:1435-1437 